\title{
Efectos del Programa Talentos en el desarrollo cognitivo y socioemocional de sus alumnos*
}

\author{
Effects of UDEC Talent Program on Cognitive and Socio-emotional Development \\ of their Participants
}

Efeitos do Programa de Talentos no desenvolvimento cognitivo e socioemocional de seus alunos

\author{
José M. Merino, ${ }^{a}$ María E. Mathiesen, ${ }^{b}$ Olga Mora, ${ }^{c}$ Ginette Castro, ${ }^{d}$ Gracia Navarro $^{e}$ \\ aDepto.de Sociología y Antropología, Facultad de Cs. Sociales, Universidad de Concepción. \\ Telf.: (41) 220-3044. Correo electrónico: jmerino@udec.cl \\ bDepto.de Sociología y Antropología, Facultad de Cs. Sociales, Universidad de Concepción. \\ Telf.: (41) 220-3150. Correo electrónico: mmathies@udec.cl \\ 'Depto. de Trabajo Social, Facultad de Ciencias Sociales, Universidad de Concepción. \\ Telf.: (41) 220-4403. Correo electrónico: omora@udec.cl \\ dDepto. de Lenguas y Traducción. Facultad de Arte y Humanidades. Universidad Católica de Temuco. \\ Telf.:(45) 220-5300. Correo electrónico: gcastro@uct.cl \\ eDepto. de Psicología, Facultad de Ciencias Sociales, Universidad de Concepción. \\ Telf.: (41) 220-3037. Correo electrónico: gnavarro@udec.cl
}

\begin{abstract}
RESUMEN
Este artículo demuestra efectos del Programa Talentos UdeC. sobre el desarrollo cognitivo y socioemocional de alumnos que asisten al menos tres años. Se comparan indicadores de desarrollo de 73 alumnos (38 en el tratamiento y 37 en el control), evaluados el 2004. El 2010 se midió vocabulario, comprensión lectora, habilidad para resolver problemas matemáticos y rendimiento académico (promedio de notas de enseñanza media, NEM y resultados de pruebas de ingreso a la universidad, PSU), como indicadores de desarrollo cognitivo. Para el desarrollo socioemocional se usaron cinco tests (motivación personal, adaptación social, responsabilidad social, conducta prosocial y comportamiento social general). Utilizando técnicas de regresión discontinua, fueron encontradas diferencias significativas a favor del grupo participante en el Programa (tratamiento), en todos los indicadores cognitivos. No fueron detectadas, sin embargo, diferencias en desarrollo socio-emocional atribuibles a las participación en la intervención. Se concluye que el programa es más eficiente en lo cognitivo.
\end{abstract}

Palabras clave: programa talentos, desarrollo cognitivo, desarrollo socio-emocional, regresión por discontinuidad.

\begin{abstract}
This paper focuses on demonstrating the impact of the UdeC Talent Program on cognitive and socioemotional development of at least three-year group of participant students. To this goal, there were compared development indicators of 73 students ( 38 treatments versus 37 controls), in 2004. In 2010, vocabulary, reading comprehension, ability for solving math problems, and academic outcomes (grades overall average at high-school (NEM), and the standardized testing battery for accessing to University, (PSU), were considered as cognitive indicators. Five tests were applied to evaluate the socioemotional development (personal motivation, social adjusting, social responsibility, prosocial behavior, and an overall social behavior scale). By applying discontinuity regression modeling, significant differences were found in favor of the Program participants (treatments), in almost all the cognitive
\end{abstract}

Este estudio exhibe resultados del Proyecto Fondecyt № 1100260 (Mathiesen, Merino, Mora, Navarro y Castro; 2010-2011). 
indicators. However, there were no significant differences in socioemotional development among treatment students. The main conclusion is that the Talent Project is more efficient and stronger in the cognitive area.

Key words: talent program, cognitive development, socio-emotional development, discontinuity regression.

\begin{abstract}
RESUMO
Demonstram-se efeitos do Programa Talentos UdeC sobre o desenvolvimento cognitivo e socioemocional de alunos assistidos pelo referido programa durante, no mínimo, três anos. Comparam-se indicadores de desenvolvimento de 73 alunos (38 em tratamento e, 37, sob controle), avaliados no ano de 2004. Em 2010, mediram-se vocabulário, compreensão de leitura, habilidade para resolver problemas matemáticos e rendimento acadêmico (a média das notas do Ensino Médio, NEM, e resultados das provas de ingresso na universidade, PSU) como indicadores de desenvolvimento cognitivo. Para avaliar o desenvolvimento socioemocional utilizaram-se cinco testes (motivação pessoal, adaptação social, responsabilidade social, conduta pró-social e comportamento social geral). Utilizando-se técnicas de regressão por descontinuidade, foram encontradas diferenças significativas favoráveis ao grupo participante no Programa (tratamento), em todos os indicadores cognitivos. Entretanto, não foram detectadas diferenças no desenvolvimento socioemocional atribuíveis à participação na intervenção. Conclui-se que o programa é mais eficiente no cognitivo.
\end{abstract}

Palavras chave: programa talentos, desenvolvimento cognitivo, desenvolvimento socioemocional, regressão por descontinuidade.

\title{
1. INTRODUCCIÓN
}

Este artículo informa los efectos producidos por el programa Talentos UdeC en diversos indicadores de desarrollo cognitivo y socioemocional en alumnos que cursaban cuarto de enseñanza media durante el 2010, integrantes de la muestra correspondiente al Proyecto Fondecyt No 1100260 (Mathiesen et al., 2010). Se espera demostrar un efecto positivo del Programa Talentos de la Universidad de Concepción en el desarrollo de los estudiantes participantes.

En el escenario social actual, que se caracteriza por la generación e intercambio vertiginoso de conocimientos, procesos económicos cada vez más flexibles y una relación de las naciones marcadas por la interdependencia y competitividad mundial (Jiménez, 2002; Mella, 2003; Hinojosa, 2004) el capital humano, esto es los conocimientos, habilidades, competencias y actitudes de sus habitantes, son decisivos para el avance económico y para la mayor competitividad de los países (Gajardo, 2003); la educación aparece como la herramienta idónea para la optimización de dicho capital, tan estrechamente relacionado con la riqueza o pobreza de las naciones y con la creación de capital social (Coleman, 1988; Jiménez, 2002; Brunner y Elacqua, 2003; Morgan \& McKerrow, 2007). La mayor parte de la interacción entre sociedad, cultura y educación, en contextos de subdesarrollo, tiene como resultado el desperdicio de talentos. Según Horowitz (1986), la diferencia entre el nivel de logro y el nivel potencial de los talentosos puede ser una buena medida del grado de aprovechamiento de los recursos de una sociedad. Por todo lo anterior se debe concordar en que los niños/as y jóvenes talentosos constituyen una reserva enorme de riqueza y que desarrollar su talento es clave para la superación de la pobreza (en Consejo Superior de Educación, 2005).

El programa Talentos UdeC., se propone satisfacer las necesidades de desarrollo cognitivo y socio-emocional en alumnos y alumnas con alto potencial intelectual. El programa está diseñado para favorecer tanto el desarrollo cognitivo, como la adaptación social del talento académico regional. Fue creado en Concepción (Chile) el año 2003; sus primeros 120 alumnos, provenientes de colegios municipales, ingresaron el año 2004. Es un programa psico-educativo de enriquecimiento extracurricular, compatible y complementario a la educación regular. Está dirigido a niños y jóvenes con talento académico, es 
financiado con aportes del Ministerio de Educación (MINEDUC), la Lotería de Concepción, las municipalidades locales y la Universidad de Concepción (UdeC). Tiene la misión de ofrecer una oportunidad educativa de excelencia para fortalecer en sus alumnos además del desarrollo cognitivo, la motivación por aprender, emprender y perseverar, la adaptación socioemocional y la responsabilidad social. Utilizando recursos académicos de la UdeC, el programa ofrece talleres y cursos disciplinarios, multidisciplinarios e instrumentales, tutorías en investigación y actividades de extensión, especialmente diseñados a partir de un modelo curricular específico.

Para ingresar a Talentos UdeC cada niño o joven participa en un proceso de selección que abarca dos etapas, en la primera los profesores, previamente capacitados, preseleccionan dos alumnos por curso en los colegios. En la segunda etapa, el programa Talentos UdeC efectúa una selección entre los alumnos preseleccionados que tienen aptitudes intelectuales sobresalientes e interés por participar. Se aplica una prueba de inteligencia general y tres pruebas de evaluación socioemocional (estas últimas no se usan en la selección).

El programa consiste en una jornada de iniciación para los alumnos, que luego participan en dos semestres académicos por año (clases viernes por la tarde y sábado por la mañana). Asimismo, en la temporada de verano, los alumnos tienen 2 semanas de clases y talleres intensivos. Por último, se ofrece una jornada de término (Feria de Resultados), en la que los alumnos exponen los resultados de sus aprendizajes al público asistente. En total el programa consta de 360 horas anuales repartidas en cinco cursos y tres talleres anuales por alumno.

La meta es formar jóvenes que mantengan y aumenten su motivación por el conocimiento, que sean propositivos, creativos, con una fuerte sensibilidad y compromiso con los demás, y se proyecten como líderes en el campo social, político y científico, entre otros. En resumen lo que se pretende es ofrecer una oportunidad efectiva para el desarrollo personal y del talento a estudiantes destacados.

La importancia de cultivar las potencialidades de los niños y niñas con talento académico y desarrollar sus habilidades sociales, así como su responsabilidad social, debe ser entendida en el contexto anterior. Teniendo en consideración lo anteriormente expuesto, así como los recursos humanos y económicos invertidos en el Programa Talentos UdeC, el propósito de este estudio se focaliza en responder la pregunta: ¿en alumnos de alto potencial intelectual, asistir al Programa Talentos UdeC tiene efectos sobre su desarrollo cognitivo y socioemocional?

\subsection{MARCO DE REFERENCIA}

Al hablar de talento, nos referimos a un término definido culturalmente que señala ciertas capacidades o habilidades socialmente deseables de algún sujeto en comparación con algún estándar, constituido generalmente por personas de edad, experiencia y medioambiente similar (Bralic y Romagnoli, 2000). Siguiendo a Gagné (2000) se puede afirmar que el talento alude al dominio destacado de habilidades, conocimientos o destrezas sistemáticamente desarrollados en al menos un campo de la actividad humana, en un grado que sitúa al individuo dentro del $10 \%$ superior del grupo de su misma edad que cultiva o ha cultivado ese campo. En el caso de nuestra cultura, es el talento intelectual o académico el que recibe mayor reconocimiento social y ha llegado a homologarse a la idea general de talento (Bralic y Romagnoli, 2000). 
Pese a esta tradición intelectualista, algunos autores han intentado ampliar la caracterización del sujeto, y en específico, del alumno talentoso o dotado, evaluando logros o habilidades potenciales en diferentes áreas: intelecto general, aptitudes académicas específicas, pensamiento creativo o productivo, habilidades de liderazgo, artes visuales o interpretativas y habilidades psicomotoras (Bralic y Romagnoli, 2000). Otros autores incluso piensan que el talento académico, además de capacidad intelectual, debería considerar un alto nivel de creatividad y un alto compromiso con las tareas o motivación para mantener un buen desempeño en éstas, de forma que el sujeto no sólo aprenda rápida y fácilmente, sino que sea original, avanzado para su edad y que genere un trabajo de importancia potencialmente duradera (Woolfolk, 1996). Respecto a esto, si bien existe relativo consenso sobre la importancia de un nivel de motivación elevado dentro de la tipificación de una persona talentosa, existe disputa respecto a la creatividad, puesto que algunos autores consideran que puede haber talento sin este componente (Bralic y Romagnoli, 2000). De hecho, Woolfolk (1996) sostiene que es posible distinguir entre el superdotado académico, que es aquel que aprende con facilidad y rapidez y obtiene buenos resultados en pruebas de inteligencia, del superdotado creativo/productivo que sobresale en situaciones que requieren una resolución de problemas de maneras novedosas y efectivas. Este segundo tipo tendría mayor probabilidad de éxito en la vida adulta.

Finalmente, un último aspecto importante en la definición de talento, es que actualmente se reconoce que éste puede mantenerse no expresado o latente, lo que significa que habría sujetos que presentarían capacidades que permiten anticipar un desempeño superior, mientras en otros esa anticipación no es posible y/o la manifestación del talento será relativamente tardía y, finalmente, habrá otros que, debido a condiciones relacionales del sujeto con las personas y su entorno, su talento no se expresará durante toda la vida, lo que pone en relieve la importancia de presentar oportunidades educativas que estimulen y permitan el desarrollo de todos los individuos (Bralic y Romagnoli, 2000).

Se ha demostrado que las capacidades destacadas como cualquier otro potencial de desarrollo en el ser humano son sólo posibilidades cuya actualización depende fundamentalmente de la experiencia y de las oportunidades que el medio les ofrezca para su desarrollo. Por tanto, esperar que estos niños se adapten a un sistema escolar que no valora sus capacidades y los obliga a ir al ritmo del promedio, puede ser perjudicial tanto para el cultivo del talento mismo como para el desarrollo integral de su personalidad (Colangelo y Davis, 1997, citado en Bralic y Romagnoli, 2000).

\section{MÉTODOS}

El diseño de la investigación es cuasi-experimental porque el grupo de tratamiento y de control no son formados aleatoriamente. El grupo-tratamiento está constituido por los alumnos que obtienen sobre 49 puntos en el Test de Matrices Progresivas Raven, aplicado el año 2004 y, por tanto, son seleccionados para participar en el Programa Talentos. El grupo-control fue formado por los alumnos y alumnas que no alcanzaron el puntaje Raven para lograr el ingreso al Programa, pero estuvieron en un rango muy cercano al puntaje de corte requerido. Los datos fueron analizados con regresión por discontinuidad porque este es un diseño cuasi-experimental, que es ampliamente utilizado en sociología y microeconomía de la educación, cuando se trata de evaluar el impacto de una intervención 
en una situación en que no existe asignación aleatoria de los individuos a los grupos de tratamiento y de control, sino específicamente los individuos son asignados al tratamiento y al control en función de un puntaje de corte (Trochim, 2001; Imbens \& Lemieux, 2007; van der Klaauw, 2008; Lee \& Lemieux, 2009; Murnane \& Willet, 2011).

En el presente estudio, la intervención consiste en pertenecer al Programa de Talentos de la Universidad de Concepción. El programa de Talentos está dirigido por la Universidad a estudiantes de enseñanza básica y media de la intercomuna de Concepción, que asisten prioritariamente a establecimientos municipales. El programa selecciona a los estudiantes talentosos en función de un proceso de doble etapa: en la primera, los profesores de los establecimientos municipales seleccionan a sus estudiantes más destacados en rendimiento académico. En la segunda etapa, el Programa Talentos de la Universidad aplica un test de Inteligencia (Matrices de Raven, 2003) sobre este conjunto de estudiantes y los jerarquiza por el puntaje obtenido en el test. Todos aquellos que, estando en quinto básico, obtienen un puntaje de 49 o superior son considerados talentosos y son invitados a participar en el programa cuando cursan sexto básico y pueden permanecer en este hasta cuarto medio. La intervención considera la realización semanal de variadas actividades académicas universitarias formales e informales (cursos, investigaciones, conferencias, discusiones, etc.).

\subsection{MUESTRA}

La muestra teórica estuvo constituida por 120 jóvenes, todos preseleccionados por el programa Talentos UdeC en el año 2004, año en el que se les aplicó el Test Raven cuando cursaban $6^{\circ}$ año básico en colegios municipales. El grupo-tratamiento se conformó con 40 estudiantes que ingresaron al Programa Talentos UdeC el 2004 y habían permanecido en él al menos tres años. Se constituyeron, además, dos grupos control, de acuerdo a sus puntajes de inteligencia: el primero quedó compuesto por 40 alumnos de inteligencia cercana al grupo tratamiento (47 y 48 puntos) y el segundo de inteligencia normal (46 a 42 puntos); este último grupo fue eliminado de los análisis que se informan en este artículo. Por lo tanto, la muestra real quedó conformada por 73 alumnos: 37 que asistieron a Talentos UdeC y por otros 36 que con inteligencia muy cercana no fueron seleccionados para el Programa. La reducción de la muestra planificada, de 80 alumnos a 73, se basa en la no participación de algunos alumnos, sea porque no aceptaron participar en el estudio actual o porque fue imposible ubicarlos por cambio de residencia o colegio, así como también, en algunos casos, porque la dirección del establecimiento no autorizó su participación.

Al momento de la medición, los jóvenes de la muestra cursaban el último año de la enseñanza media o secundaria (92\%). Su promedio de edad fue de 17.5 años, mayoritariamente de 18 ó 17 años, con el 52 y 46\% respectivamente. El 55\% eran hombres y el $45 \%$ mujeres. La mayoría asistía a Liceos científico-humanistas (62\%), el resto a Liceos comerciales $(21 \%)$ o a Liceos técnicos (18\%). En relación al tipo de sostenedor del establecimiento educacional, durante el año 2004 todos los alumnos asistían a colegios municipales; durante el 2010 la mayoría seguía perteneciendo a colegios municipales $(56 \%)$, pero un $22 \%$ asistía a colegios particulares subvencionados y el $19 \%$ restante a colegios de la Corporación Educacional de la Cámara de la Construcción, mientras un $3 \%$ asistía a establecimientos particulares no subvencionados. 
En relación a la inteligencia de los integrantes de la muestra, su promedio fue de 49,6; este puntaje está entre el percentil 90 y el 95 del baremo general de 1993 (Raven, 2003) para niños/as entre 10 y 11 años, edad de estos alumnos cuando se les evaluó la inteligencia. El margen de variación estuvo entre el percentil 75 y el 99. La desviación estándar, como era de esperar, muestra una variación moderada, puesto que la muestra, como ya dijimos, consideró sólo los alumnos del Programa Talentos UdeC y el grupo control de inteligencia cercana, excluyendo los integrantes del grupo control II de inteligencia normal. Estamos conscientes que estos datos sobre inteligencia corresponden a la obtenida por estos jóvenes en el año 2004 y que durante los seis años transcurrido pueden haber variaciones diferenciales en los individuos de la muestra. Sin embargo, esas variaciones, de existir, deben ser capturadas en el grupo-control y descontadas por tanto de los efectos atribuibles al Programa Talentos.

\subsection{INSTRUMENTOS UTILIZADOS}

A continuación, se describen brevemente los instrumentos que se aplicaron para medir distintos aspectos del desarrollo cognitivo.

Test de Matrices Progresivas (Raven, 2003). El test es una adaptación realizada en Argentina en el año 1993. A través del instrumento se mide el nivel de inteligencia mediante la presentación de 60 láminas de dibujos a las que les falta una porción. Estas imágenes están distribuidas en cinco series con doce dibujos cada una; existen seis y ocho alternativas de respuesta de entre las que se debe elegir la que corresponde. Las series van aumentando en complejidad progresivamente. El puntaje máximo alcanzable es de 60 puntos, ya que cada respuesta correcta recibe un punto. El test permite ubicar el percentil que le corresponde al evaluado por edad en tablas especialmente diseñadas.

Test de Vocabulario en Imágenes, TEVI-R (Echeverría, Herrera \& Segure, 2009). Este test de vocabulario en imágenes mide el nivel de vocabulario pasivo en niños y jóvenes; es de amplia utilización debido a que es de fácil y rápida aplicación, la que puede ser grupal o individual. Es un instrumento que se encuentra estandarizado en el ámbito chileno y permite comparar por edad los resultados de un individuo con una norma establecida. La tarea a resolver por el niño o joven es indicar una de cuatro láminas que se le presentan, de acuerdo al estímulo auditivo del entrevistador.

Puntajes Prueba de Selección Universitaria (PSU) y Notas de Enseñanza Media (NEM). La PSU es una prueba que consta de varias partes, dos de ellas obligatorias, a saber, matemáticas y lenguaje, y que es requisito para el ingreso a las universidades chilenas tradicionales. Los resultados de estas pruebas son públicos y se dan a conocer a través de Internet. Para efectos de la investigación se han considerado los puntajes tanto de matemáticas y lenguaje, como el puntaje ponderado asignado por concepto de promedio general de Notas de la Enseñanza Media (NEM).

A continuación, se describen brevemente los instrumentos usados para evaluar algunos aspectos del Desarrollo Socioemocional:

-Cuestionario de Adaptación Socioemocional. Se trata de una escala tipo Likert, construida a por el equipo Talentos UdeC. La escala original formada por 27 ítems, con 5 alternativas de respuesta (totalmente en desacuerdo hasta totalmente de acuerdo). Posterior a un estudio que demostró características psicométricas adecuadas (Mathiesen et al., 2011), se suprimieron 4 ítems, por tanto la escala quedó con 23 ítems distribuidos en 
tres subescalas: Habilidades emocionales, con 10 ítems; Percepción de autoeficacia, con 7; y Habilidades sociales, con 5. Del total de 13 ítems invertidos del formato original, quedaron 10 en la escala final.

-Cuestionario de Motivaciones e Intereses. Este instrumento también fue elaborado por el equipo de sicólogos del Programa Talentos UdeC. Se trata de una escala Likert de 28 ítems, sobre los que el sujeto debe manifestar su grado de acuerdo desde el total acuerdo (5 puntos) hasta el total desacuerdo (1 punto). Se hizo un estudio psicométrico que demuestra la confiabilidad de este cuestionario, con un alfa de Cronbach de $0.88 \mathrm{y}$ correlaciones altamente significativas de todos sus ítems con el total de la escala.

-Cuestionario de Comportamientos Socialmente Responsables. Se trata de un listado de 19 comportamientos que muestran responsabilidad social sobre los que el sujeto debe informar la frecuencia con que los realiza desde nunca (0) hasta siempre (4). También se hizo un estudio psicométrico de este cuestionario que demuestra su confiabilidad, con un alfa de Cronbach de 0.82 y correlaciones altamente significativas de todos sus ítems con el total de la escala.

-Teenage Inventory of Social Skills (TISS) de Inderbitzen and Foster (1992). Este Inventario de Conductas Sociales, informa adecuada confiabilidad y validez para USA (Inderbitzen y Clavin, 1992). La versión en español se tomó de Roa (2010). Se trata de un cuestionario de tipo Likert de 40 afirmaciones: 20 de conductas prosociales y 20 antisociales, sobre las que el encuestado debe manifestar en qué grado desarrolla esos comportamientos en el colegio, desde nada (1 punto) hasta muchas veces (5), pasando por poco, a veces y varias veces con 2,3 y 4 puntos, respectivamente. Revirtiendo los ítems, la escala puede usarse con los 40 ítems como de conducta prosocial total y/o como una prosocial y otra antisocial, cada una de 20 ítems. Con el estudio psicométrico realizado se encontró alfas de Cronbach de 0.78 para la escala total y para la de conducta antisocial, mientras que para la de conducta prosocial fue de 0.85 .

-Texas Social Behavior Inventory (Helmreiech, Stapp \& Ervin, 1974 en Robinson y Shaver, 1991). Este cuestionario de comportamiento social fue traducido y adaptado por el equipo investigador. El instrumento tiene dos formatos: en este caso se aplicó el formato A, que tiene 16 ítems, 6 de ellos invertidos. Los sujetos deben manifestar en qué medida los representan una serie de afirmaciones, desde No me representa para nada (1 punto) hasta Me representa completamente (5 puntos) pasando por No mucho, Algo y Bastante con 2, 3 y 4 puntos respectivamente. Es un instrumento corto, de fácil comprensión y respuesta. El estudio psicométrico realizado mostró una confiabilidad adecuada con un alfa de 0.77 y solo un ítem que no correlacionó significativamente con el total.

\subsection{PROCEDIMIENTO}

Durante julio y agosto del año 2010, se obtuvieron las autorizaciones de los Directores de establecimientos, a partir de agosto y hasta noviembre, después de la consecusión de los asentimientos de los alumnos y de los consentimientos de los padres, se recolectaron las notas de enseñanza media de los registros de los establecimientos y se aplicaron los test en salas facilitadas por el colegio; cuando esto no fue posible, se tomaron en dependencias de la Universidad de Concepción. Se aplicaron 3 pruebas de desarrollo cognitivo y 5 de desarrollo socioemocional. En enero de 2011, de los listados oficiales de Internet, se obtuvieron los puntajes PSU y NEM. Luego se construyó una base de 
datos SAS utilizando el programa correspondiente (Statistical Analysis System, versión 9.1.2; Licencia Universidad de Concepción, Escuela de Graduados, SITE 050543001). Con esta base se programaron y se analizaron los datos que se presentan en este trabajo. Previo a las regresiones por discontinuidad, fue efectuado un análisis de normalidad de las variables, determinándose que a pesar de las particularidades de la muestra, las variables se comportaban como normales o muy cercanas.

\section{RESULTADOS}

Las siguientes tablas presentan el análisis e interpretación de las ecuaciones de regresión por discontinuidad. Como en otras versiones de diseños contrafácticos de inferencia causal, en regresión por discontinuidad se requiere un modelo estadístico que incluya un término para el pretest, otro para el posttest y una variable con codificación dummie para representar la pertenencia al programa (Trochim, 2001; Murnane \& Willet, 2011).

En este estudio, el pretest estará representado por la variable usada como puntaje de corte, esto es, el puntaje del test Raven para cada estudiante centrado en el promedio aritmético. La variable posttest corresponde a la variable dependiente del modelo, esto es, las variables de desarrollo cognitivo y/o socioemocional que han sido medidas al estudiante individual. La variable utilizada para representar la pertenencia al grupo-tratamiento y al grupo-control es el dummy (0/1), en que el 1 es asignado al grupo-tratamiento y 0 al grupo-control.

Después de transformar el pretest (centramiento), se debe examinar detenidamente las relaciones entre la variable pretest y la variable posttest en el modelo, a fin de determinar el grado polinomial necesario en la ecuación, en función del número de curvaturas en la distribución conjunta. Cuando no hay puntos curvos en la distribución se está frente a un modelo lineal. La regla de oro es modelar dos órdenes de polinomiales más altos que lo indicado por los puntos de inflexión detectados en la distribución pretest-posttest (Trochim, 2001; Lee \& Lemeieux, 2009). Por tanto, si la relación bivariada pretestposttest es lineal (sin líneas curvas), se debería comenzar modelando una polinomial de segundo-orden. Esto garantiza una sobre-identificación del modelo, lo que se requiere para estimar sin sesgos el efecto del Programa. Dado que el modelo está sobre-identificado, sin embargo, la estimación es ineficiente porque incluye términos no necesarios, lo que aumenta artificialmente los errores estándar y conduce a subestimación de los efectos de la intervención.

Sobre la base anterior, el modelo se debe refinar para remover sucesivamente términos innecesarios y re-estimar los efectos del tratamiento con mayor eficiencia. Se debe comenzar examinando los términos polinomiales cuadráticos con coeficientes no significativos, eliminando esos términos innecesarios del modelo. Enseguida, se debe proceder de la misma manera con las interacciones lineales hasta lograr un modelo parsimonioso y de la más alta eficiencia e insesgamiento en el establecimiento del efecto del tratamiento. En este estudio todas las relaciones pre-post resultaron lineales por lo que se trabajó -como punto de partida- con un modelo de polinomiales cuadráticas. En todos los casos se eliminaron del modelo final los términos cuadráticos e interactivos por ausencia de significación estadística, por lo que todos los modelos lineales finales, que exponemos a continuación, constituyen estimaciones sin sesgos y eficientes del efecto. 
En primer lugar, presentaremos los resultados de las regresiones por discontinuidad de los aspectos cognitivos de los alumnos del Programa Talentos y de los del grupo control de inteligencia cercana. Luego, se presentarán los resultados obtenidos en el desarrollo socioemocional.

\subsection{DIMENSIONES COGNITIVAS}

En la Tabla 1 se presentan los resultados obtenidos para las diferencias entre el grupo de intervención y de control en los puntajes finales de las notas de enseñanza media. Estos puntajes indican el efecto que tiene sobre el rendimiento académico global de los estudiantes y su participación en la intervención, esto es, en el Programa Talentos de la UdeC. Se observa que la tabla de regresión indica un intercepto (574.7 puntos); un puntaje Raven_Cut (-18.1) y el predictor Tratamiento (104.2 puntos). Los valores que participan en la interpretación son el intercepto y el coeficiente de regresión estimado para el predictor Tratamiento. El puntaje Raven_Cut es la variable utilizada como criterio de asignación (Raven), centrada en el puntaje de corte (49), para ordenar bajo 49 los valores del grupo control y sobre 49 los valores del grupo tratamiento. Aunque no se utiliza directamente en la interpretación, hace posible la asignación automática de los casos a la variable Tratamiento, que es la que hace la estimación correcta del efecto de la intervención.

Como se ha establecido, el valor del coeficiente de regresión de la variable dummy Tratamiento estima el efecto de la intervención comparando el valor del intercepto (promedio de puntajes NEM de los estudiantes pertenecientes al grupo-control) respecto del valor que debe ser agregado a dicho promedio para estimar el promedio de puntajes NEM en el grupo-tratamiento. La interpretación es que el efecto de la intervención -participar en el Programa de Talentos de la UdeC- eleva en 104.2 puntos el valor del promedio de notas NEM para el estudiante talentoso promedio. Esto significa que el estudiante que participa en el programa Talentos, en promedio tiene 104.2 puntos más que el estudiante que no participa en el programa Talentos. Esa diferencia -como lo demuestra la significación de la diferencia del T-Student en la ecuación- es significativa al 2 por ciento. Es decir, participar en el Programa Talentos eleva el promedio final de notas del estudiante de enseñanza media (NEM) en algo más de 100 puntos. Esto significa -contrafácticamenteque los estudiantes participantes en el programa Talentos UdeC deberían haber esperado, en promedio, 104 puntos menos en puntaje promedio de enseñanza media si -debido a la asignación en función del puntaje RAVEN- hubiesen pertenecido al grupo-control del estudio, esto es, no hubiesen tenido la posibilidad de acceder al Programa Talentos. Esto habla de la importancia de pertenecer al Programa Talentos como factor predictor de alto desarrollo académico terminal en el ciclo de estudios de enseñanza media. Si consideramos que este factor predictor está, además, altamente involucrado en el acceso y términos de estudios universitarios, podemos tener una clara idea del importante impacto que el Programa Talentos UdeC -como intervención de este estudio- tiene sobre el desarrollo de los estudiantes. 
Tabla 1. Regresión por discontinuidad del efecto del Programa Talentos sobre el Promedio de las Notas de Enseñanza Media (NEM) de sus participantes

\begin{tabular}{|c|c|c|c|c|c|}
\hline \multicolumn{6}{|c|}{ Parámetros Estimados } \\
\hline Variable & Gl & $\begin{array}{c}\text { Coeficiente } \\
\text { Regresión }\end{array}$ & $\begin{array}{c}\text { Error } \\
\text { Standard }\end{array}$ & $\begin{array}{c}\text { Valor } \\
\text { T Student }\end{array}$ & $\operatorname{Pr}>\mathrm{T}$ \\
\hline Intercepto & 1 & 574.69 & 23.32 & & .0001 \\
\hline Raven-Cut & 1 & -18.11 & 11.44 & -1.6 & .1186 \\
\hline Tratamiento & 1 & 104.19 & 44.46 & 2.34 & .0223 \\
\hline
\end{tabular}

Un segundo indicador de rendimiento cognitivo de los estudiantes fue medido, en este estudio, mediante la comparación de los rendimiento generales de los estudiantes en los años 2007, 2008 y 2009, los tres últimos años en que esta cohorte del programa Talentos UdeC se desempeña en enseñanza media. Este variable, denominada promedio general 2007-2009, pondera las notas de los estudiantes en los tres años para obtener una nota promedio general, en la escala 1 a 7 . En ese sentido, es un indicador de rendimiento cognitivo general cercano a NEM y debería tener consistencia con los valores entregados por éste, constituyéndose en un verdadero recurso de validación convergente para las Notas de Enseñanza Media (NEM) de cada estudiante.

La Tabla 2, más abajo expuesta, indica convergentemente con la situación encontrada para las notas de enseñanza media (NEM), que los alumnos del grupo expuesto al Programa Talentos UdeC tienen más de medio punto (0.524) de ventaja, en promedio, que los alumnos que no asistieron al programa y que aquí se concentran en el grupo control.

Tabla 2. Regresión por discontinuidad del efecto del Programa Talentos sobre el promedio general de rendimiento académico de sus participantes en los años 2007 al 2009

\begin{tabular}{|c|c|c|c|c|c|}
\hline \multicolumn{6}{|c|}{ Parámetros Estimados } \\
\hline Variable & Gl & $\begin{array}{c}\text { Coeficiente } \\
\text { Regresión }\end{array}$ & $\begin{array}{c}\text { Error } \\
\text { Standard }\end{array}$ & $\begin{array}{c}\text { Valor } \\
\text { T Student }\end{array}$ & $\operatorname{Pr}>\mathrm{T}$ \\
\hline Intercepto & 1 & 5.7318 & 0.1046 & 54.78 & .0001 \\
\hline Raven-Cut & 1 & -0.0639 & 0.0535 & -1.19 & .2365 \\
\hline Tratamiento & 1 & 0.5243 & 0.2038 & 2.57 & .0123 \\
\hline
\end{tabular}

El cuadro anterior expresa que mientras el grupo-control tuvo una nota general promedio de 5.73 entre los años 2007 al 2009, el grupo que asistió en el período al Programa Talentos UdeC, obtiene 6.25 como nota promedio general, en el mismo período, diferencia que con un error de $1.23 \%$, permite concluir que asistir al programa Talentos incrementa el rendimiento promedio general de esos estudiantes.

Los siguientes párrafos exponen el análisis e interpretación de la ecuación de regresión por discontinuidad. En la Tabla 3 se exhiben los efectos de la participación en el Programa Talentos sobre la diferencia en puntajes de PSU-Matemáticas, en el grupo tratado respecto del grupo control. El efecto de la intervención -participar en el Programa de Talentos de 
la UdeC- eleva en 113.15 puntos el valor del puntaje de la prueba PSU-Matemáticas para el estudiante talentoso promedio. Esa diferencia -como lo demuestra la significación del T-Student en la ecuación- es significativa al 9 por diez mil. Es decir, se ha detectado una diferencia de puntajes tan alta a favor de los participantes en el Programa de Talentos, que sólo 9 veces en diez mil experimentos se produciría un resultado diferente. Estas conclusiones expresan bien la importancia del programa Talentos sobre el desarrollo cognitivo matemático de este grupo de estudiantes. Participar en el Programa Talentos eleva el puntaje del estudiante en la PSU-Matemáticas en más de 110 puntos, lo que indica que la intervención facilita enormemente el acceso de estos estudiantes a la Universidad y pronostica con gran exactitud el éxito posterior en los estudios universitarios.

Tabla 3. Regresión por discontinuidad del efecto del Programa Talentos sobre el promedio de sus participantes en Puntajes de PSU Matemáticas

\begin{tabular}{|c|c|c|c|c|c|}
\hline \multicolumn{6}{|c|}{ Parámetros Estimados } \\
\hline Variable & Gl & $\begin{array}{c}\text { Coeficiente } \\
\text { Regresión }\end{array}$ & $\begin{array}{c}\text { Error } \\
\text { Standard }\end{array}$ & $\begin{array}{c}\text { Valor } \\
\text { T Student }\end{array}$ & Pr $>$ T \\
\hline Intercepto & 1 & 524.73 & 17.02 & 30.8 & .0001 \\
\hline Raven-Cut & 1 & -17.17 & 8.28 & -2.1 & .0423 \\
\hline Tratamiento & 1 & 113.15 & 32.52 & 3.5 & .0009 \\
\hline
\end{tabular}

En la misma forma en que fue medido directamente el promedio general de los estudiantes, como se expresó en la Tabla 2, y sus resultados fueron utilizados para corroborar los efectos de las Notas de Enseñanza Media (NEM), en la Tabla 4 se presenta el efecto del programa Talentos sobre el rendimiento general en matemáticas de los alumnos, medidos entre el año 2007 y 2009. Esto constituye una forma de ratificar empíricamente los importantes hallazgos de la Tabla 3. Como puede observarse en la Tabla 4, la regresión discontinua indica que el grupo control tiene un promedio de matemáticas entre el año 2007 y 2009 igual a 5.47, en la escala 1 a 7 . El promedio en el grupo tratado en el mismo periodo es 0.61 puntos mayor, esto es, igual a 6.08. La diferencia en promedio en matemática en los tres años es, como expresa la ecuación de regresión discontinua en la Tabla 4, significativa al $2.6 \%$, valor dos veces inferior al máximo permitido en ciencias sociales $(\mathrm{p}<.05)$.

Esto significa que un segundo criterio diferente de validación empírica de los efectos de Talentos UdeC sobre el rendimiento matemático, ratifica las conclusiones extraídas desde la Tabla 3.

Tabla 4. Regresión por discontinuidad del efecto del Programa Talentos sobre el promedio 20072009 en rendimiento en Matemáticas de sus participantes

\begin{tabular}{|c|c|c|c|c|c|}
\hline \multicolumn{6}{|c|}{ Parámetros Estimados } \\
\hline Variable & Gl & $\begin{array}{c}\text { Coeficiente } \\
\text { Regresión }\end{array}$ & $\begin{array}{c}\text { Error } \\
\text { Standard }\end{array}$ & $\begin{array}{c}\text { Valor } \\
\text { T Student }\end{array}$ & $\operatorname{Pr}$ T \\
\hline Intercepto & 1 & 5.4711 & 0.1382 & 39.64 & .0001 \\
\hline Raven-Cut & 1 & -0.0998 & 0.0706 & -1.41 & .1622 \\
\hline Tratamiento & 1 & 0.6108 & 0.2692 & 2.27 & .0265 \\
\hline
\end{tabular}


Un tercer criterio de validación empírico del importante efecto detectado en este estudio del Programa Talentos UdeC sobre la formación matemática de sus participantes, es un test de habilidades cuantitativas desarrollado por este equipo investigador, utilizando como base pruebas de ensayo PSU Matemáticas y aplicado posteriormente a los participantes en el estudio. El test incluyó doce preguntas de habilidad cuantitativa que arrojaron un Alpha de Cronbach de 0.74 .

La Tabla 5 muestra los resultados obtenidos para el test de habilidades y conocimientos matemáticos, cuando fueron aplicados los procedimientos de regresión discontinua. El grupo de tratamiento -los estudiantes participantes en el Programa Talentos UdeCobtienen $29.84 \%$ más respuestas correctas que los estudiantes pertenecientes al grupo contrafáctico o control. Mientras el grupo-tratamiento logra $60.8 \%$ de respuestas correctas en el test, el grupo-control sólo obtiene 30.9\%. Esto demuestra que las habilidades cognitivas-cuantitativas -expresadas en respuestas correctas a los problemas planteados en el grupo-tratado- casi duplican las respuestas de ese tipo obtenidas en el grupo-control. Pero lo más importante es que esa diferencia tiene una probabilidad inferior a 3 veces en mil, de atribuirse al azar: es efecto directo de la exposición del grupo-tratado a las intervenciones desarrolladas en el Programa Talentos.

Debe enfatizarse que hemos mostrado evidencia de tres pruebas independientes que conducen a demostrar el importante impacto del Programa Talentos sobre la dimensión cuantitativa del rendimiento académico de estudiantes de enseñanza media.

Tabla 5. Regresión por discontinuidad del efecto del Programa Talentos sobre la habilidad cuantitativa y conocimientos matemáticos de sus participantes

\begin{tabular}{|c|c|c|c|c|c|}
\hline \multicolumn{6}{|c|}{ Parámetros Estimados } \\
\hline Variable & Gl & $\begin{array}{c}\text { Coeficiente } \\
\text { Regresión }\end{array}$ & $\begin{array}{c}\text { Error } \\
\text { Standard }\end{array}$ & $\begin{array}{c}\text { Valor } \\
\text { T Student }\end{array}$ & $\operatorname{Pr}>\mathrm{T}$ \\
\hline Intercepto & 1 & 0.3099 & 0.0499 & 6.20 & .0001 \\
\hline Raven-Cut & 1 & -0.0418 & 0.0255 & -1.64 & .1062 \\
\hline Tratamiento & 1 & 0.2984 & 0.0971 & 3.07 & .0030 \\
\hline
\end{tabular}

Como puede observarse en el cuerpo de la Tabla 6, el coeficiente de regresión indica que el grupo tratamiento tiene en promedio 82.1 puntos más que el grupo control. Esto indica que asistir al Programa Talentos tiene también efectos positivos sobre los resultados PSU de Ciencias. Sin embargo, la significación estadística de esta diferencia es residual y menor a las anteriores puesto que rechaza sólo al 5.7\% la hipótesis nula que establece la inexistencia de diferencias entre grupo tratado y control. Sin embargo, mantiene el sentido positivo de los efectos detectados para el promedio final de las notas de enseñanza media (NEM) y de los descritos para la prueba PSU de Matemáticas y habilidad cuantitativa. Lo importante es demostrar que el Programa Talentos en cuanto intervención educacional es capaz de generar en forma consistente cambios cognitivos entre sus participantes en dimensiones de rendimiento académico acumulado en un período de cuatro años, así como en desarrollo de habilidades cuantitativas medida en el test estandarizado nacional más importante y que también se expresa en el test estandarizado nacional de ciencias, más relevante del sistema educacional nacional. 
Tabla 6. Regresión por discontinuidad del efecto del Programa Talentos sobre el promedio de sus participantes en Puntajes de PSU Ciencias

\begin{tabular}{|c|c|c|c|c|c|}
\hline \multicolumn{6}{|c|}{ Parámetros Estimados } \\
\hline Variable & Gl & $\begin{array}{c}\text { Coeficiente } \\
\text { Regresión }\end{array}$ & $\begin{array}{c}\text { Error } \\
\text { Standard }\end{array}$ & $\begin{array}{c}\text { Valor } \\
\text { T Student }\end{array}$ & $\operatorname{Pr}$ T \\
\hline Intercepto & 1 & 509.70 & 22.59 & 22.6 & .0001 \\
\hline Raven-Cut & 1 & -11.06 & 10.42 & -1.06 & .2941 \\
\hline Tratamiento & 1 & 82.06 & 42.16 & 2.0 & .0575 \\
\hline
\end{tabular}

En cuanto a las dimensiones cognitivas relacionadas con las áreas humanistas y del lenguaje, los efectos del Programa Talentos UdeC no son tan nítidos como en el área cuantitativa y se han detectado sólo algunos efectos positivos muy específicos. En particular cuando se ha construido una medida combinada de los promedios de la asignaturas de lenguaje en los años 2007 al 2009 y se ha examinado como la regresión por discontinuidad compara los promedios de los grupos tratamientos y control en ese índice compuesto, se ha detectado efectos significativos de la exposición asociada al Programa Talentos. En la Tabla 7, se observa que el promedio de lenguaje entre los años 2007-2009, para los participantes del grupo tratamiento es de 6.16 puntos, mientras que el mismo promedio en el grupo control es de 5.45 puntos. Lo importante es que la diferencia entre ambos promedio tiene una significación estadística de $2.7 \%$, lo que indica que no se puede atribuir al azar, sino a la exposición a la intervención, esto es, la asistencia al Programa Talentos, que es la única distinción importante que presentan ambos grupos contrafácticos.

Tabla 7. Regresión por discontinuidad del efecto del Programa Talentos sobre el promedio de sus participantes en notas de lenguaje en el período 2007-2009

\begin{tabular}{|c|c|c|c|c|c|}
\hline \multicolumn{6}{|c|}{ Parámetros Estimados } \\
\hline Variable & Gl & $\begin{array}{c}\text { Coeficiente } \\
\text { Regresión }\end{array}$ & $\begin{array}{c}\text { Error } \\
\text { Standard }\end{array}$ & $\begin{array}{c}\text { Valor } \\
\text { T Student }\end{array}$ & $\operatorname{Pr}$ T \\
\hline Intercepto & 1 & 5.457 & 0.162 & 33.69 & .0001 \\
\hline Raven-Cut & 1 & -0.077 & 0.082 & -0.94 & .3518 \\
\hline Tratamiento & 1 & 0.712 & 0.315 & 2.26 & .0272 \\
\hline
\end{tabular}

Con el propósito de establecer los impactos del Programa Talentos en el desarrollo cognitivo en el área humanista fue también utilizado el Test TEVI, instrumento estandarizado que mide el nivel de vocabulario pasivo entre los estudiantes del estudio. La Tabla 8 presenta los resultados que fueron obtenidos con el modelo de regresión discontinua para los puntajes en el test TEVI. Los estudiantes que participaron en el grupo tratamiento puntuaron en promedio 103.7 en el test TEVI, mientras que los estudiantes adscritos al grupo-control puntuaron en promedio 89.5. Aun cuando el grupo-tratamiento supera en 14.2 puntos al grupo-control, esa diferencia es significativa con un $7.7 \%$ de nivel de error, lo que es $2.7 \%$ más alto que el máximo permitido de $5 \%$. La conclusión es que aunque el grupo-tratado es mejor que el control en los puntajes de Vocabulario, la diferencia es sólo residual y no conclusiva. 
Tabla 8. Regresión por discontinuidad del efecto del Programa Talentos sobre el puntaje de sus participantes en el Test Estandarizado de Vocabulario Pasivo (TEVI)

\begin{tabular}{|c|c|c|c|c|c|}
\hline \multicolumn{6}{|c|}{ Parámetros Estimados } \\
\hline Variable & Gl & $\begin{array}{c}\text { Coeficiente } \\
\text { Regresión }\end{array}$ & $\begin{array}{c}\text { Error } \\
\text { Standard }\end{array}$ & $\begin{array}{c}\text { Valor } \\
\text { T Student }\end{array}$ & $\operatorname{Pr}>$ T \\
\hline Intercepto & 1 & 89.52 & 4.08 & 21.94 & .0001 \\
\hline Raven-Cut & 1 & -1.900 & 2.09 & -0.91 & .3657 \\
\hline Tratamiento & 1 & 14.21 & 7.93 & 1.79 & .0775 \\
\hline
\end{tabular}

En cuanto a los resultados de la regresión por discontinuidad en los casos de la PSU Lenguaje, de la PSU Historia y del Test de Comprensión Lectora (tablas que no se presentan en el texto), el grupo tratamiento en los tres casos obtiene puntajes promedios superiores a los obtenidos por su contrafáctico en el grupo control, sin embargo, en todas las situaciones esas diferencias de puntaje promedio no alcanzan significación estadística. Llama la atención que el efecto del Programa Talentos UdeC no tenga la misma intensidad en el área del desarrollo humanístico y del lenguaje que el detectado en el área científica o basado en razonamiento cuantitativo. En el PSU Lenguaje, en PSU Historia y en Comprensión Lectora, las diferencias entre el grupo tratado y control logran significación estadística al $\mathrm{p}>.18$ y $\mathrm{p}>.14$ y $\mathrm{p}>.20$, respectivamente, esto es, entre tres y cuatro veces el máximo de error permitido en la docimacia de hipótesis de la corriente principal en ciencias sociales.

\subsection{DIMENSIONES SOCIO-EMOCIONALES}

Además del estímulo directo de las dimensiones principales del área cognitiva en los participantes, otro de los propósitos esenciales del Programa Talentos UdeC es contribuir a mejorar aspecto claves del área psicosocial o socioemocional de los estudiantes que participan en sus actividades. La evaluación del impacto del Programa debe, por tanto, incluir estas dimensiones subjetivas de manera similar a como se hizo con las dimensiones principales del desarrollo cognitivo.

Diez indicadores de desarrollo socio-emocional fueron seleccionados y medidos para evaluar el impacto potencial del programa en este contexto. El ajuste o adaptación del estudiante fue ponderado mediante cuatro dimensiones: una medida de adaptación global, otra de adaptación social, de adaptación socio-emocional y, finalmente, mediante una valoración de la autoeficacia. El comportamiento del estudiante fue ponderado mediante el Inventario Adolescente de Habilidades Sociales (TISS), instrumento internacional de amplia reputación, que me permite medir simultáneamente conducta prosocial, conducta antisocial y conducta general. Se midió, además, la responsabilidad social de los estudiantes, la motivación personal y, finalmente, el Inventario de Conducta Social-Texas, que permite establecer conducta social adolescente.

Todos los instrumentos presentaron adecuada calidad de medida, tanto en confiabilidad como en validez, y tenían suficiente aplicación internacional, con la excepción de la escala de responsabilidad social y de motivación personal, que fueron proporcionados por psicólogos del equipo profesional del Programa Talentos UdeC. 
Tabla 9. Diferencias de promedios entre grupo-tratamiento y grupo-control en variables socioemocionales del estudio, establecidas mediante regresión por discontinuidad

\begin{tabular}{|c|c|c|c|c|}
\hline Variables Socioemocionales & $\begin{array}{c}\text { Grupo } \\
\text { Tratamiento }\end{array}$ & $\begin{array}{c}\text { Grupo } \\
\text { Control }\end{array}$ & T Test & $\begin{array}{c}\text { P } \\
\text { Value }\end{array}$ \\
\hline Adaptación general & 4.06 & 3.77 & 1.39 & .17 \\
\hline Adaptación social & 4.05 & 4.02 & 0.13 & .85 \\
\hline Adaptación emocional & 3.50 & 3.12 & 1.49 & .14 \\
\hline Adaptación eficacia & 4.32 & 3.99 & 1.60 & .11 \\
\hline Comportamiento general (TISS) & 3.79 & 3.71 & 0.50 & .55 \\
\hline Conducta prosocial & 3.71 & 3.86 & 0.20 & .83 \\
\hline Conducta antisocial & 2.16 & 2.33 & 0.87 & .38 \\
\hline Responsabilidad social & 2.49 & 2.43 & 0.33 & .74 \\
\hline Motivación & 4.38 & 4.15 & 1.18 & .24 \\
\hline Comportamiento social (TEXAS) & 3.69 & 3.48 & 0.96 & .34 \\
\hline
\end{tabular}

La característica principal que destaca en las dimensiones socio-emocionales que se exponen en la Tabla 9 es que ninguna presenta diferencias significativas inferiores al 5\%. Esto implica que el Programa Talentos no logro impactar en ninguna de estas dimensiones psicosociales de manera importante y estadísticamente sostenible. Aun cuando en casi todos los casos, el grupo-tratamiento (participantes en el Programa) obtuvo puntajes o promedios superiores a los del grupo contrafáctico (control), en ninguno la diferencia resultó siquiera ser marginalmente cercana a la significación estadística. La escala de autoeficacia $(\mathrm{p}>.11)$ y la de adaptación emocional $(\mathrm{p}>$.14) son las más cercanas y están dos y tres veces, respectivamente, sobre el máximo de error permitido en las ciencias sociales.

La evidencia anterior significa que el propósito de impactar en dimensiones psicosociales del estudiante, es un objetivo en 'el Programa Talentos' que no está siendo exitoso y debería ser reformulado. Existe la posibilidad también que la instrumentación seleccionada para medir las dimensiones socio-emocionales que exhibe la Tabla 9 no hayan sido las adecuadas para capturar el efecto que el Programa, que en estas dimensiones efectivamente logra.

Dos argumentos deberían ser opuestos a esta última afirmación. En primer lugar, al cambiar la ventana (el ancho de los grupos bajo y sobre el corte de la variable-asignación) para expandir las diferencias entre los grupos de tratamiento y control, no mejoran el efecto de ninguna variable, pese a aumentar la diferencia de puntajes Raven entre los estudiantes del grupo-tratado respecto del grupo-control. En segundo lugar, al examinar el comportamiento de las escalas con trayectoria internacional respecto de aquellas cuya experiencia es más bien local, no se observa ninguna diferencia que pudiese sugerir alguna tendencia a obtener mejores resultados con otras escalas.

La evidencia indica, en consecuencia, que todo el éxito que el Programa Talentos UdeC logra en las dimensiones cognitivas cuantitativas, no se repite en las áreas humanísticas ni en las socio-emocionales, aspectos que tal vez ameritan algunos ajustes en 
las metodologías, técnicas o prácticas instruccionales, utilizadas en el Programa para producir los cambios buscados.

\section{CONCLUSIÓN}

Este estudio tenía como propósito establecer la existencia de diferencias cognitivas y socioemocionales -atribuibles a la participación en Talentos UdeC en estudiantes que asistieron al programa- cuando son comparados con estudiantes del mismo nivel escolar, contexto social y residencial de estudiantes que no asistieron al programa. El diseño del estudio fue cuasi-experimental y, basado en el modelo de resultados potenciales de Rubin, utilizó regresión discontinua como estrategia analítica, para comparar estudiantes de enseñanza media de niveles similares y cercanos en talento (en cuanto medido por el Test de Inteligencia Matrices de Raven). En función del puntaje obtenido en el test Raven, un grupo de estudiantes de enseñanza media fue invitado a participar en el Programa Talentos UdeC. El puntaje de corte fue 49 puntos en el mencionado test. Los estudiantes que obtuvieron un puntaje igual o mayor a 49 puntos conformaron el grupo-tratamiento de este estudio. Los estudiantes bajo este corte fueron parte del grupo-control. El Programa Talentos UdeC -en la cohorte medida en este estudio- fue una intervención que comenzó el año 2004 e incluye estudiantes con al menos tres años de participación en el Programa. El Programa Talentos UdeC, en cuanto intervención educacional, se basó en exponer al grupo participante a un conjunto sistemático de actividades académicas en la Universidad de Concepción, consistente en asistencia a cursos, charlas, conferencias, actividades de investigación, conocimiento de la vida universitaria, discusiones, paneles, etc. Todas estas actividades fueron planificadas y ejecutadas institucionalmente por docentes universitarios de la U. de Concepción, quienes han organizado, dirigido e implementado el Programa Talentos desde sus inicios. Las mediciones finales del estudio, en ambos grupos (tratado y control), fueron hechas en el transcurso del año 2010.

Las evidencias encontradas en este estudio establecen fehacientemente la existencia de diferencias en rendimiento estudiantil, especialmente en el área cuantitativa, que favorecen a los niños y jóvenes que asistieron al Programa: los resultados en matemáticas, en notas de enseñanza media, en promedio general de notas 2007-2009, en promedio en asignaturas de matemáticas en el período 2007-2009, en promedio en asignatura de lenguaje en el período 2007-2009 y, finalmente, en el test estandarizado de medición de Vocabulario, TEVI-R. En todas estas dimensiones cognitivo-cuantitativas (con la excepción de lenguaje y vocabulario), los estudiantes del grupo-tratamiento del estudio -esto es, aquellos que participan en el Programa Talentos UdeC- lograron puntajes significativamente más altos que su grupo contrafáctico, estudiantes en el grupo-control. Esto indica que los más altos puntajes obtenidos por estos alumnos se deben a su participación en el Programa Talentos y no al azar: si los estudiantes pertenecientes al grupo control hubiesen participado, en cambio, en el programa Talentos, sus puntos serían los que la regresión discontinua estimó para el grupo tratado.

Sin embargo, los efectos del Programa Talentos UdeC no son destacables en el área cognitiva-humanista ni entre las dimensiones socio-emocionales o psicosociales. No fueron detectadas diferencias significativas - provenientes de la pertenencia al Programa- entre los estudiantes de los grupos estudiados en las mediciones estandarizadas asociadas al 
Lenguaje (PSU-L), como tampoco en la medición estandarizada asociada a la Historia (PSU-H). Llama la atención, además, que ninguna dimensión socio-emocional o psicosocial medida entre los estudiantes presentara efectos atribuibles a la participación en Talentos UdeC. Fue medida la adaptación general, social y emocional; la autoeficacia; el comportamiento general (TISS); la conducta prosocial y antisocial; la responsabilidad social; la motivación individual y el comportamiento social (TEXAS). Ninguna de estas escalas, la mayoría con amplia aplicación y prestigio internacional, demostró que el programa Talentos UdeC haya tenido efectos comprobables empíricamente sobre el desarrollo socio-emocional de los estudiantes.

Varias son las razones que pueden dar cuenta de los perfiles enunciados en los párrafos anteriores. En primer lugar, el éxito del Programa en lo cognitivo-cuantitativo, especialmente en las magnitudes (tamaño de efectos) y dimensiones específicas (Notas de Enseñanza Media, NEM; promedios generales en matemáticas, promedios de PSUMatemáticas y Ciencias), parece indicar que los niños y niñas de educación básica expresan preferencias sistemáticas por cursos relacionados con ciencias en general y/o matemáticas $\mathrm{y}$, prácticamente, no eligen las ofertas programáticas del área de humanidades, que sólo comienzan a ser atractivos cuando los alumnos cursan enseñanza media. En segundo lugar, lo anterior también podría explicar la ausencia de cambios en dimensiones socio-emocionales. Habría poco énfasis en estas áreas en la intervención o al menos menor que en las anteriores. Aunque subsiste también la hipótesis que el desarrollo socio-emocional es de más lenta maduración y, por ende, no se expresaría tan cerca del período de intervención.

Aún una última reflexión en esta dirección, indicaría que es conveniente evaluar otros indicadores de desarrollo socio-emocional, puesto que la calidad de la medición en esta área del crecimiento individual, no tiene la misma evidencia o respaldo que la exhibida por el desarrollo cognitivo-cuantitativo. Es posible que otros instrumentos socio-emocionales puedan mostrar mejor cuán felices, satisfechos, interesados y autónomos, se manifiestan estudiantes talentosos cuando participan en programas como Talentos UdeC. En estudios futuros se debería enfrentar esta duda incluyendo mediciones de otros aspectos de la psicología social de los jóvenes.

\section{REFERENCIAS BIBLIOGRÁFICAS}

Bralic, S., \& Romagnoli, C. (2000). Niños y jóvenes con talento: una educación de calidad para todos. Santiago de Chile: Dolmen.

Brunner, J.J. y Elacqua, G. (2003). Informe Capital Humano en Chile. Santiago de Chile: Universidad Adolfo Ibáñez, Escuela de Gobierno.

Coleman, J. (1988). Social Capital in the Creation of Human Capital. Cambridge, Masachussetts and London: The Beknap Press of Harvard University Press.

Consejo Superior de Educación. (2005). Relaciones entre Instituciones de Educación Superior y la Enseñanza Escolar. Santiago: MINEDUC.

Echeverría, M., Herrera, M., y Segure, J. (2002). Test de Vocabulario en Imágenes (TEVI- R). ( $3^{\circ}$ Ed. rev.). Chile: Editorial Universidad de Concepción.

Gagné, F. (2000). Un modelo diferenciador de dotación y talento. Notas Personales. Traducidas por Sonia Bralic (con autorización del autor). Montreal: Université du Québec à Montreal.

Gajardo, M. (2003). Globalización, sociedad del conocimiento y nuevas tecnologías en educación: desafíos para una mejor gestión. Visiones de la Educación, vol. 3, 29-33. 
Hinojosa, M. (2004). La sociedad del conocimiento en el contexto nacional (Chile). Visiones de la Educación, vol. 5, 21-32.

Horowitz, FD., O'Brien, M. (1986). Gifted and talented children: State of knowledge and directions for research. American Psychologist, vol. 41, n. 10, 1147-1152.

Imbens, G. y Lemieux, T. (2007). Regression Discontinuity Designs: A Guide to Practice. Working Paper 13039. National Bureau of Economic Research (NBER). April 2007.

Inderbitzen, H. y Clavin, H. (1992). An investigation of the Construct Validity of de Teenage Inventatory of Social Skills: A Covergent Approach. Paper presentado en la Junta anual de la Asociación por el Avance de la Terapia de Comportamiento, Boston, USA.

Inderbitzen, H. y Foster, S. (1992). The Teenage Inventory of Social Skills: Development, Realibility, and Validity. Psychological Assesment, vol. 4, n. 4, 451-499.

Jiménez, J. (2002). Las tareas de la gestión escolar. En C. Figueroa y,J. Jiménez (Eds.), Globalización, sociedad del conocimiento y nuevas tecnologías en educación: desafios hacia una mejor gestión (pp. 153-169). Valparaíso: CENLADEC.

van der Klauuw, W. (2008). Breaking the Link between Poverty and Low Student Achievement: An Evaluation of Title I. Journal of Econometrics, vol. 142, n. 2, 731-756.

Lee, D. L. y Lemieux, T. (2009). Regression Discontinuity Designs in Economics. Working Paper 14723. National Bureau of Economic Research (NBER). February 2009.

Mathiesen, M. E., Merino, J.M., Mora, O., Navarro, G. y Castro, G. (2010-2011). Incubando capital humano regional: evaluación longitudinal de un programa extracurricular universitario para estudiantes de establecimientos municipales de alto potencial académico. Proyecto Fondecyt $\mathrm{N}^{\circ} 1100260$.

Mathiesen, M. E., Castro, G., Merino, J. M., Mora, O. y Navarro, G. (2011). Adaptación socioemocional de niños/as y jóvenes: Evaluación de un instrumento nuevo en estudiantes de la provincia de Concepción. Estudios Pedagógicos. vol. 37, n. 2, 61-75.

Mella, E. (2003). La Educación en la sociedad del conocimiento y el riesgo. Revista Enfoques Educacionales, vol. 5, n.1, 107-114.

Morgan, S. y Mckerrow, M. (2007). Human Capital Development and Frontiers of Research in Sociology of Education. En Garnett Picot, R. Saunders y A. Sweetman (Eds.), Fulfilling Potencial, Creating Success: Perspectives on Human Capital Development (pp. 85-225). Montreal: McGill-Queen's University Press.

Murnane, RJ. y Willet, JB. (2011). Methods Matter. Improving Causal Inference in Educational and Social Science Research. New York: Oxford University Press.

Raven, J.C. (2003). Test de Matrices Progresivas: Escala General. Buenos Aires: Paidós.

Roa, R. (2010). Influencia de la conducta prosocial en el desarrollo educativo y social de alumnos de octavo básico de la comuna de Concepción. Memoria inédita para obtener el título de Sociólogo. Universidad de Concepción.

Robinson, J. y Shaver, P. (1991). Measures of personality and social psycological attitudes. San Diego, California. Academic Press.

Trochim, W.M. (2001). The Research Methods Knowledge Base. Second Edition. Cincinnati, OH: Atomicdogpublishing.

Woolfolk, A. E. (1996). Psicología Educativa. (7a. Edición). México: Prentice Hall. 